\title{
Step instability and island formation during annealing of pseudomorphic InGaAs/GaAs layers
}

\author{
A. Riposan, G. K. M. Martin, and J. Mirecki Millunchick ${ }^{\text {a) }}$ \\ Department of Materials Science and Engineering, University of Michigan, 2300 Hayward, \\ Ann Arbor, Michigan 48109-2136
}

(Received 12 May 2003; accepted 4 October 2003)

\begin{abstract}
The morphological stability of compressively strained $\mathrm{In}_{0.27} \mathrm{Ga}_{0.73} \mathrm{As} / \mathrm{GaAs}$ pseudomorphic layers has been investigated during annealing. Large three-dimensional islands form at the beginning of annealing on initially flat surfaces, likely to relieve strain energy. The islands disappear with increasing annealing, being reabsorbed into the terraces. At the same time, the step line destabilizes forming cusps that inject two-dimensional vacancy islands into the terrace. At high temperatures, this process leads to a severe deterioration of the morphology that is not due to decomposition. The island dissolution and the development of the step instability are likely alternative paths towards the reduction of surface energy. (C) 2003 American Institute of Physics. [DOI: 10.1063/1.1631053]
\end{abstract}

III-V compound semiconductor alloys are used in the fabrication of optoelectronic and microelectronic devices, most of which require flat surfaces and interfaces. However, the surface morphology of strained layers varies widely. It has been shown that the strain energy of lattice-mismatched layers can be relieved through the formation of threedimensional (3D) islands, both during growth and annealing. ${ }^{1,2}$ In addition, strain is known to cause step bunching and mounding. ${ }^{3}$ Changes in the step structure occur even in the absence of mismatch strain to minimize the surface energy. ${ }^{4,5}$ This picture is complicated by surface segregation and desorption, which affect the surface energetics of ternary alloy layers. ${ }^{6,7}$ Although previous work has examined the morphological stability during film growth, the stability of pseudomorphic layers in the absence of the deposition flux is equally important. This study investigates the morphological stability of InGaAs pseudomorphic layers during annealing. Our results show that for compressively strained layers, initially flat surfaces destabilize upon annealing, with the severe deterioration of the step structure and the formation of pits.

All films were grown using a molecular beam epitaxy chamber, with solid sources for $\mathrm{Ga}$, In, and a valved cracking cell for $\mathrm{As}_{4}$ with $T_{\text {cracker }}=600^{\circ} \mathrm{C}$. The $\mathrm{As}_{4}$ beam equivalent pressure (BEP) was measured prior to growth using a beam flux monitor. The growth rates for In and Ga were calibrated using reflection high-energy electron diffraction (RHEED) intensity oscillations. The GaAs (001) substrates were prepared by heating to $T \approx 600^{\circ} \mathrm{C}$ under As overpressure to remove the oxide layer. After oxide desorption, GaAs buffers were grown at $T \approx 575^{\circ} \mathrm{C}$ and $\mathrm{BEP} \approx 12 \times 10^{-6}$ Torr. Pseudomorphic $\operatorname{In}_{0.27} \mathrm{Ga}_{0.73} \mathrm{As}$ films (misfit strain $\approx 1.9 \%$ ) were grown in the layer-by-layer growth mode to a thickness $h=25$ monolayers (ML), at $T=487^{\circ} \mathrm{C}$ and $\mathrm{BEP}=16$ $\times 10^{-6}$ Torr, and a growth rate of $R=0.77 \mathrm{ML} / \mathrm{s}$. After growth, the layers were either quenched or annealed under various conditions $\left(460<T<538^{\circ} \mathrm{C}\right.$ and $6 \times 10^{-6}<\mathrm{BEP}$ $<16 \times 10^{-6}$ Torr). The quenching was performed by rapidly

${ }^{a)}$ Electronic mail: joannamm@umich.edu cooling the sample under As overpressure, which minimizes the diffusion during cooling and preserves the gross surface morphology. The samples were examined ex situ by tapping mode atomic force microscopy (AFM). Number density, area coverage and aspect ratio for surface features were calculated from the AFM micrographs, using several $5 \times 5 \mu \mathrm{m}$ scans for each sample. The surface composition was examined by $\mathrm{x}$-ray photoelectron spectroscopy (XPS).

Figure 1 shows the morphological evolution of $h$ $=25 \mathrm{ML} \mathrm{In}_{0.27} \mathrm{Ga}_{0.73} \mathrm{As} / \mathrm{GaAs}$ layers, grown at $T=487^{\circ} \mathrm{C}$ and $\mathrm{BEP}=16 \times 10^{-6}$ Torr, and annealed at $T=487^{\circ} \mathrm{C}$ and $\mathrm{BEP}=6 \times 10^{-6}$ Torr. The surface reconstruction during growth and annealing remained a weak $(2 \times 3)$, as observed by RHEED. No relaxation of the in-plane lattice parameter was observed during either growth or annealing. At the end of growth [Fig. 1(a)], the morphology consists of wide flat terraces with an average width $\approx 400 \mathrm{~nm}$, and wavy step edges, due to the partial incorporation of two-dimensional (2D) islands. Also present is a large density of 2D islands, consistent with layer-by-layer growth. The islands are typically $1 \mathrm{ML}$ in height; however, small $2 \mathrm{ML}$ high islands are also observed occasionally.

Upon short annealing time, large 3D islands form on top of the initially flat alloy surface [Fig. 1(b)]. These features are referred to as "mega-islands" to distinguish them from the typical strain-relieving islands that form during growth above the critical thickness. ${ }^{8}$ The height of the mega-islands in these films varies between 2 and $4 \mathrm{ML}$, while the basal dimensions are on the order of several hundred nanometers. Mega-islands up to $8 \mathrm{ML}$ tall were observed during annealing under higher As overpressures (not shown). The megaislands are elongated along the $[1 \overline{1} 0]$ direction and their tops consist of a wide (001) facet.

Both the 2D islands and the mega-islands disappear with increasing annealing time. Figure 2 shows the total volume per square micrometer taken up by $2 \mathrm{D}$ islands and megaislands during annealing at $\mathrm{BEP}=6 \times 10^{-6}$ Torr and 16 $\times 10^{-6}$ Torr. For both overpressures, the total volume accumulated by the $2 \mathrm{D}$ islands decreases to negligible values at long annealing. In contrast, the total volume of material ac- 
(a)

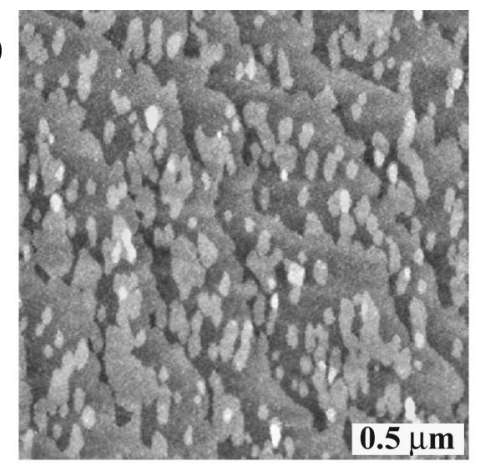

(b)

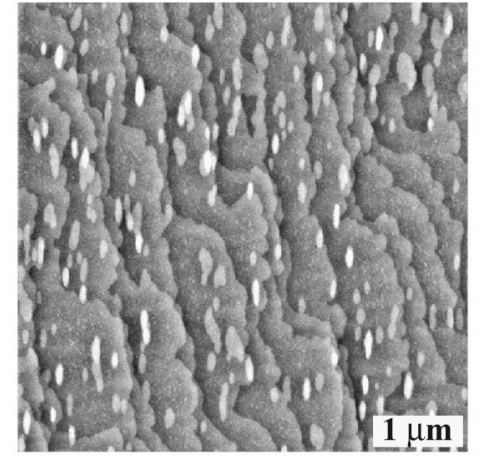

(c)

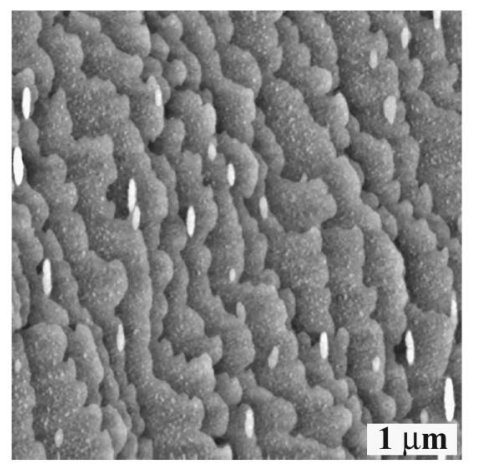

(d)

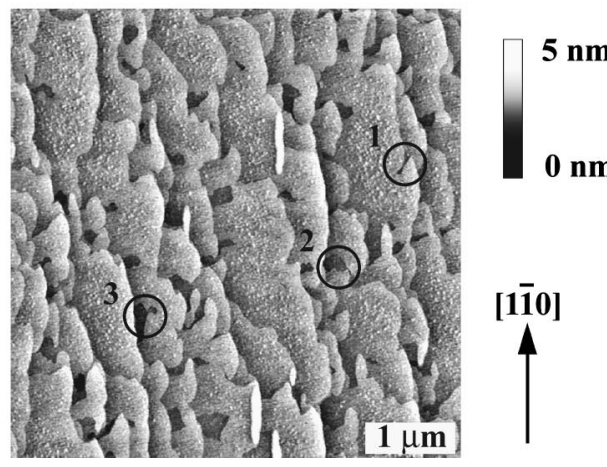

FIG. 1. AFM images showing the surface morphology of 25-ML-thick $\mathrm{In}_{0.27} \mathrm{Ga}_{0.73} \mathrm{As} / \mathrm{GaAs}$ layers, (a) grown at $T=487^{\circ} \mathrm{C}$ and $\mathrm{BEP}=16$ $\times 10^{-6}$ Torr, and annealed at $\mathrm{BEP}=6 \times 10^{-6}$ Torr for (b) 5 , (c) 10 , and (d) $25 \mathrm{~min}$

cumulated in mega-islands reaches a pronounced peak at short annealing times. This peak is higher at $\mathrm{BEP}=16$ $\times 10^{-6}$ Torr, presumably due to decreased diffusion lengths. ${ }^{9}$ The maximum total volume reached by the megaislands is higher than the available volume of $2 \mathrm{D}$ islands, suggesting that the growth of the mega-islands occurs not only at the expense of the $2 \mathrm{D}$ islands, but also from additional sources of material, such as the step edges.

The total volume of mega-islands decreases at longer annealing times, indicating that the reduction in their density is not due entirely to coalescence, in which case the total

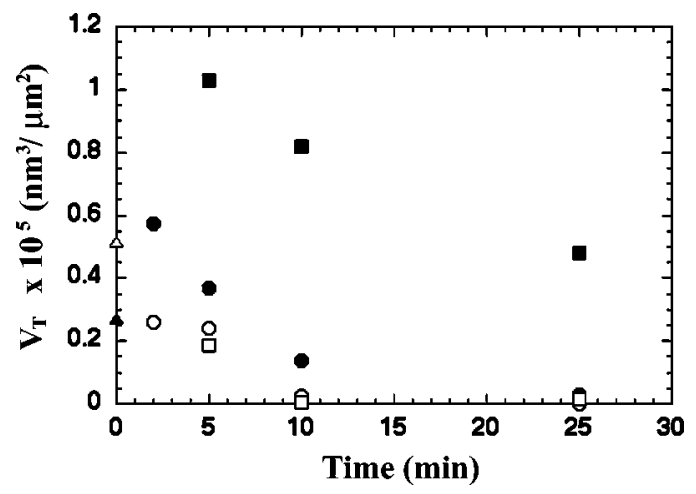

FIG. 2. Total volume per square micrometer of 2D islands (open symbols) and mega-islands (filled) vs the annealing time for $h=25 \mathrm{ML}$ $\mathrm{In}_{0.27} \mathrm{Ga}_{0.73} \mathrm{As} / \mathrm{GaAs}$ layers, at $T=487^{\circ} \mathrm{C}$ and $\mathrm{BEP}=6 \times 10^{-6}$ Torr (circles) and $16 \times 10^{-6}$ Torr (squares). Data at time $=0$ (triangles) represents the asgrown condition, where the closed triangle represents the bilayer islands present on the as-grown surface.

volume would remain roughly constant. Moreover, the basal area of mega-islands increases with time, suggesting that these features do not simply evaporate during annealing. Rather, the 3D mega-islands grow initially, and are reabsorbed into the terraces. During this process, atoms detach from the upper layers and climb down, leading progressively to a decrease in feature height and an increase in its basal area. The increase in the basal area suggests that the climbing-down rate of adatoms is higher than the detachment rate from the island. The in-plane aspect ratio of the megaislands increases strongly during annealing, indicating a high anisotropy in the climbing down rate.

In addition to the formation of the metastable megaislands, the step structure of these alloy films also exhibits an unusual behavior during annealing. The step roughness decreases initially, as expected. ${ }^{10}$ However, an instability appears with increasing annealing time, leading to the development of a pronounced undulation in the step line. This results in the formation of cusps which penetrate into the step edge [Fig. 1(c)] leading to the formation of 2D pits [Fig. 1(d)]. The injection of pits via step cusping occurs through several consecutive stages, which can all be observed in various regions of the micrograph in Fig. 1(d). First, the cusps sharpen and penetrate into the step (1); subsequently, the cusp tip blunts and widens, forming a "bay" that advances into the terrace (2). Finally, the edges of the bay close behind, leaving a two-dimensional vacancy island inside the terrace (3). For these annealing conditions, the wavelength of the instability and the resulting vacancy island separation is on the order of $\sim 300 \mathrm{~nm}$. Step bunching is also observed at long annealing times due to the step-step interaction in compressed layers. ${ }^{3}$

The step destabilization is intensified at higher annealing temperatures. Figure 3 shows the morphology of a $h$ $=25 \mathrm{ML}$ film grown at $T=495^{\circ} \mathrm{C}$ and $\mathrm{BEP}=16$ $\times 10^{-6}$ Torr, and annealed for $10 \mathrm{~min}$ at $T=505^{\circ} \mathrm{C}$ and $\mathrm{BEP}=12 \times 10^{-6}$ Torr. In this case, the step structure is highly fragmented, consisting of a large number of smaller overlapping terraces and a high density of intersecting edges. A small number of mega-islands are present on this surface, as well as multimonolayer deep pits formed at the intersection of several terrace fragments. 


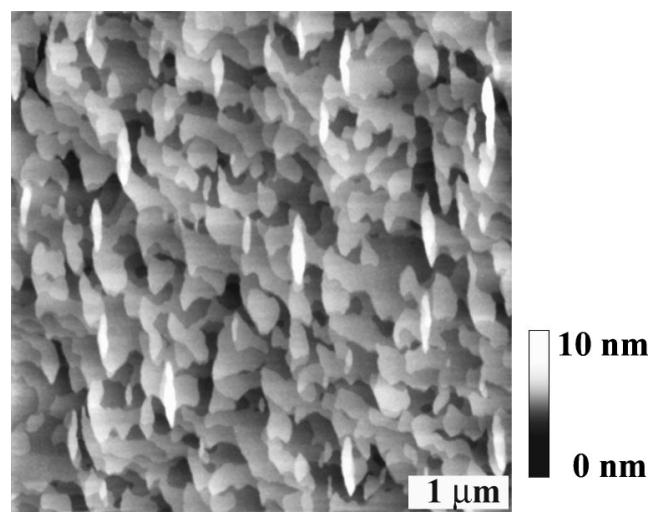

FIG. 3. AFM image showing the surface morphology of an $h=25 \mathrm{ML}$ $\mathrm{In}_{0.27} \mathrm{Ga}_{0.73} \mathrm{As} / \mathrm{GaAs}$ layer, grown at $T=495^{\circ} \mathrm{C}$ and $\mathrm{BEP}=16 \times 10^{-6}$ Torr, and annealed at $T=505^{\circ} \mathrm{C}$ and $\mathrm{BEP}=12 \times 10^{-6}$ for $10 \mathrm{~min}$.

Both the formation of mega-islands and the destabilization of steps have been observed in these experiments over a wide range of annealing conditions $(6<\mathrm{BEP}<16$ $\times 10^{-6}$ Torr and $460<T<540^{\circ} \mathrm{C}$ ). These unusual phenomena may be understood considering the interplay between the strain and the surface energies of this system during annealing. Previous annealing experiments on pseudomorphic $\mathrm{SiGe/Si}$ films with similar misfit strain resulted in the formation of the 3D island-pit morphology which develops during growth to relieve strain. ${ }^{2}$ That morphology has not been observed for the annealing of InGaAs alloys, possibly due to the increased surface energy under an As overpressure. Also, the critical adatom concentration for nucleation of 3D islands might not be reached in the InGaAs system in the absence of a deposition flux. Preliminary results show that few megaislands form during the annealing of thinner layers with the same composition, suggesting that the formation of these features is also strain-driven. ${ }^{11}$ The amount of strain relieved by mega-islands is likely small, considering their rather short and wide shape.

Despite the fact that the mega-islands relieve some strain at the beginning of annealing, they eventually disappear. This is not likely due to coalescence nor to evaporation. A possible driving force for mega-island dissolution is the minimization of surface energy of the alloy. Measurements of the surface composition by XPS show that the indium concentration at the surface reaches $\sim 58 \%$ at the end of growth, higher than the bulk composition due to In segregation, and decreases to $\sim 36 \%$ after $25 \mathrm{~min}$ annealing at $T=487^{\circ} \mathrm{C}$. Because the surface energy of GaAs is higher than that of InAs, ${ }^{12,13}$ a decrease in the surface In concentration leads to an increase in the surface energy of the alloy. The dissolution of the mega-islands, which is accompanied by a decrease in surface area (and hence a decrease in surface energy), occurs to balance the effect of the changing surface composition. In addition, the mega-islands are expected to be enriched in In due to strain relaxation. ${ }^{14}$ As the In becomes depleted from the mega-islands, the driving force for islanding decreases, thus contributing further to their disappearance.

The cusping of the steps and the formation of vacancy islands are not likely due to the decomposition of the surface, since the In surface concentration remains higher than the intended bulk composition. Therefore, the destabilization of the step structure is likely in response to changes in the surface and/or strain energies. It has been shown that undulating steps can reduce the surface energy compared with surfaces with straight steps, and they become unstable when the wavelength reaches a critical value. ${ }^{5}$ The development of such an instability results in the morphology observed in our experiments. In addition, calculations show that steps on compressive surfaces have a negative line energy, such that the overall surface energy is reduced through the creation of a new step line. ${ }^{15,16}$ This favors the development of the instability, since the length of the step edges increases during the cusping process. Thus, the step instability competes with the formation and the dissolution of mega-islands as mechanisms of energy reduction.

In conclusion, pseudomorphic $\mathrm{In}_{0.27} \mathrm{Ga}_{0.73} \mathrm{As} / \mathrm{GaAs}$ (001) layers were grown and annealed to study their morphological stability. It was found that these surfaces destabilize upon annealing, with the formation and dissolution of large 3D islands, as well as the formation of cusps in the step edges. The cusps inject $2 \mathrm{D}$ vacancies into the terraces and lead to the deterioration of the step structure. The islands form at the beginning of annealing to relieve some strain energy, but dissolve as the surface is annealed. These phenomena are likely in response to a reduction of the surface energy with increasing annealing time.

The authors gratefully acknowledge useful discussions with Professor B. G. Orr, Professor C. Pearson, and Professor M. L. Falk, and the support of the NSF Grant No. DMR00 92602.

${ }^{1}$ D. J. Eaglesham and M. Cerullo, Phys. Rev. Lett. 64, 1943 (1990).

${ }^{2}$ D. E. Jesson, K. M. Chen, S. J. Pennycook, T. Thundat, and R. J. Warmack, Phys. Rev. Lett. 77, 1330 (1996).

${ }^{3}$ J. Tersoff, Y. H. Phang, Z. Zhang, and M. G. Lagally, Phys. Rev. Lett. 75, 2730 (1995).

${ }^{4}$ O. L. Alerhand, D. Vanderbilt, R. D. Meade, and J. D. Joannopoulos, Phys. Rev. Lett. 61, 1973 (1988).

${ }^{5}$ J. Tersoff and E. Pehlke, Phys. Rev. Lett. 68, 816 (1992).

${ }^{6}$ J. M. Moison, C. Guille, F. Houzay, F. Barthe, and M. Van Rompay, Phys. Rev. B 40, 6149 (1989).

${ }^{7}$ C. Heyn, Phys. Rev. B 66, 075307 (2002).

${ }^{8}$ A. Riposan, G. K. M. Martin, M. Bouville, M. L. Falk, and J. Mirecki Millunchick, Surf. Sci. 525, 222 (2002).

${ }^{9}$ B. J. Riel, K. Hinzer, S. Moisa, J. Fraser, P. Finnie, P. Piercy, S. Fafard, and Z. R. Wasilewski, J. Cryst. Growth 236, 145 (2002).

${ }^{10}$ R. E. Caflisch, E. Weinan, M. F. Gyure, B. Merriman, and C. Ratsch, Phys. Rev. E 59, 6879 (1999).

${ }^{11}$ A. Riposan, G. K. M. Martin, and J. Mirecki Millunchick (unpublished).

${ }^{12}$ N. Moll, A. Kley, E. Pehlke, and M. Scheffler, Phys. Rev. B 54, 8844 (1996).

${ }^{13}$ E. Pehlke, N. Moll, A. Kley, and M. Scheffler, Appl. Phys. A: Mater. Sci. Process. A65, 525 (1997).

${ }^{14}$ T. Walther, C. J. Humphreys, and A. G. Cullis, Appl. Phys. Lett. 71, 809 (1997).

${ }^{15}$ Y. H. Xie, G. H. Gilmer, C. Roland, P. J. Silverman, S. K. Buratto, J. Y. Cheng, E. A. Fitzgerald, A. R. Kortan, S. Schuppler, M. A. Marcus, and P. H. Citrin, Phys. Rev. Lett. 73, 3006 (1994).

${ }^{16}$ V. M. Kaganer and K. H. Ploog, Phys. Rev. B 64, 205301 (2001). 